\title{
Performance Measurement of the microPET Focus 120 Scanner
}

\author{
Jin Su Kim ${ }^{1,2}$, Jae Sung Lee ${ }^{1,2}$, Ki Chun $\mathrm{Im}^{3}$, Su Jin Kim ${ }^{1,2}$, Seog-Young Kim³, Dong Soo Lee ${ }^{1,2}$, and Dae Hyuk Moon ${ }^{3}$ \\ ${ }^{1}$ Department of Nuclear Medicine, Seoul National University College of Medicine, Seoul, Korea; ${ }^{2}$ Interdisciplinary Programs in \\ Radiation Applied Life Science Major, Seoul National University College of Medicine, Seoul, Korea; and ${ }^{3}$ Department of Nuclear \\ Medicine, University of Ulsan College of Medicine, Asan Medical Center, Seoul, Korea
}

The microPET Focus 120 scanner is a third-generation animal PET scanner dedicated to rodent imaging. Here, we report the results of scanner performance testing. Methods: $A{ }^{68} \mathrm{Ge}$ point source was used to measure energy resolution, which was determined for each crystal and averaged. Spatial resolution was measured using a ${ }^{22} \mathrm{Na}$ point source with a nominal size of 0.25 $\mathrm{mm}$ at the system center and various off-center positions. Absolute sensitivity without attenuation was determined by extrapolating the data measured using an ${ }^{18} \mathrm{~F}$ line source and multiple layers of absorbers. Scatter fraction and counting rate performance were measured using 2 different cylindric phantoms simulating rat and mouse bodies. Sensitivity, scatter fraction, and noise equivalent counting rate (NECR) experiments were repeated under 4 different conditions (energy window, 250 750 $\mathrm{keV}$ or $350 \sim 650 \mathrm{keV}$; coincidence window, 6 or $10 \mathrm{~ns}$ ). A performance phantom with hot-rod inserts of various sizes was scanned, and several animal studies were also performed. Results: Energy resolution at a $511-\mathrm{keV}$ photopeak was $18.3 \%$ on average. Radial, tangential, and axial resolution of images reconstructed with the Fourier rebinning (FORE) and filtered backprojection (FBP) algorithms were 1.18 (radial), 1.13 (tangential), and $1.45 \mathrm{~mm}$ full width at half maximum (FWHM) (axial) at center and 2.35 (radial), 1.66 (tangential), and $2.00 \mathrm{~mm}$ FWHM (axial) at a radial offset of $2 \mathrm{~cm}$. Absolute sensitivities at transaxial and axial centers were $7.0 \%$ (250 750 keV, $10 \mathrm{~ns}), 6.7 \%$ (250 750 keV, $6 \mathrm{~ns})$, $4.0 \%$ (350 650 keV, $10 \mathrm{~ns}$ ), and 3.8\% (350 650 keV, $6 \mathrm{~ns}$ ). Scatter fractions were $15.9 \%$ (mouse phantom) and $35.0 \%$ (rat phantom) for $250 \sim 750 \mathrm{keV}$ and $6 \mathrm{~ns}$. Peak NECR was 869 $\mathrm{kcps}$ at $3,242 \mathrm{kBq} / \mathrm{mL}$ (mouse phantom) and $228 \mathrm{kcps}$ at 290 $\mathrm{kBq} / \mathrm{mL}$ (rat phantom) at $250 \sim 750 \mathrm{keV}$ and $6 \mathrm{~ns}$. Hot-rod inserts of $1.6-\mathrm{mm}$ diameter were clearly identified, and animal studies illustrated the feasibility of this system for studies of whole rodents and mid-sized animal brains. Conclusion: The results of this independent field test showed the improved physical characteristics of the F120 scanner over the previous microPET series systems. This system will be useful for imaging studies on small rodents and brains of larger animals.

Received Feb. 12, 2007; revision accepted May 31, 2007.

For correspondence or reprints contact: Jae Sung Lee, PhD, Department of Nuclear Medicine, Seoul National University College of Medicine, 28 YungunDong, Chongno-Gu, Seoul 110-744, Korea.

E-mail: jaes@snu.ac.kr

COPYRIGHT @ 2007 by the Society of Nuclear Medicine, Inc.
Key Words: small-animal PET; performance measurement; instrumentation; molecular imaging; small-animal imaging

J Nucl Med 2007; 48:1527-1535

DOI: 10.2967/jnumed.107.040550

$\mathbf{P}$ ET is a noninvasive, diagnostic imaging technique that produces functional or biochemical images of a body (1-4). Although PET is regarded as a powerful translational research tool between animal models and human clinical applications (5-8), clinical PET scanners used for human studies do not have satisfactory spatial resolution or sensitivity for studies of small animals, such as rats and mice (9-11). Because the linear dimensions of organs in these animals are 10 or more times smaller in each dimension than in human subjects, the spatial resolution of a suitable PET scanner must be similarly higher and the voxel size should also be reduced according to sampling theory $(9,10)$. To obtain images of the same quality as in humans, the sensitivity of such high-resolution PET scanners must also be increased, as the number of counts per voxel is inversely proportional to voxel volume. Therefore, detector ring diameter and crystal element size must be reduced to increase photon-detection efficiency and spatial resolution.

The microPET Focus 120 (F120) is a third-generation microPET scanner dedicated to animal imaging and was developed by Siemens Medical Solutions, Inc. It features higher light collection efficiency, better optical isolation between adjacent elements, and a denser detector block packing fraction than previously released microPET series with equivalent detector ring diameters and axial field of view (FOV) (12). Although the physical characteristics of this unit were reported previously at a conference (13), to our knowledge, no full performance measurement results by independent users have been reported in the literature. Here, we report the results of independent field tests on the system performance of the F120, including its spatial resolution, sensitivity, scatter fraction, and counting rate performance. In addition, the utility of this system for animal PET studies is discussed. 


\section{MATERIALS AND METHODS}

\section{System Description}

F120 is a ring-type, high-sensitivity, high-resolution PET scanner intended for imaging small animals, such as rats, mice, and cats. Its detector array consists of 96 detector blocks around a circle such that the face-to-face diameter of the ring is $15 \mathrm{~cm}$. Each detector module is placed in time coincidence with 7 opposite modules to give an effective transaxial FOV of approximately $10 \mathrm{~cm}$ and an axial FOV of $7.6 \mathrm{~cm}$. Each detector module consists of a $12 \times 12$ array of lutetium oxyorthosilicate (LSO) crystals of size $1.5 \times 1.5 \times 10 \mathrm{~mm}$. The LSO end is optically coupled to a position-sensitive photomultiplier tube via an $8 \times 8$ bundle of optical fibers.

PET data are acquired in list mode, and list-mode data can be sorted into 3-dimensional (3D) sinograms using different span numbers and ring differences or directly into 2-dimensional (2D) sinograms by single-slice rebinning (SSRB) (14). Images can be reconstructed directly from the 3D sinograms using 3D orderedsubset expectation maximization (OSEM 3D), a 3D reprojection algorithm (3DRP), or a 3D maximum a posteriori (MAP) (15-17). Filtered backprojection (FBP) or 2D ordered-subset expectation maximization (OSEM 2D) can be applied to 2D sinograms resorted by SSRB or a Fourier rebinning algorithm (FORE) (18).

We compare the specification of this scanner with those of a previous microPET system with the same ring diameter (microPET R4; R4) and with another Focus scanner with a larger diameter (microPET Focus 220; F220) (Table 1) $(12,19)$.

\section{Energy Resolution}

Crystal energy spectra within an energy window of $100 \sim 811$ $\mathrm{keV}$ were obtained. Events were acquired using a ${ }^{68} \mathrm{Ge}$ point source for $600 \mathrm{~s}$ in energy-spectrum mode.

Energy resolution was determined for each crystal in the system and calculated as full width at half maximum (FWHM) of a 511$\mathrm{keV}$ energy peak divided by the energy peak value $(\Delta \mathrm{E} / \mathrm{E}=$ FWHM/E). The mean value of all crystal energy resolutions was calculated.

\section{Spatial Resolution}

Spatial resolution was measured using a ${ }^{22} \mathrm{Na}$ point source with a nominal diameter of $0.25 \mathrm{~mm}$, embedded in a lucite disk (Isotope Products Laboratories). The activity of the point source was $673.4 \mathrm{kBq}(18.2 \mu \mathrm{Ci})$. Spatial resolution measurements were not corrected for source dimension, positron range, or acolinearity of positron annihilation $(12,20)$.

The point source was placed on the microPET Focus imaging bed and centered in transaxial and axial FOVs. Data were acquired for $1 \mathrm{~min}$ in list mode with an energy window of $250 \sim 750 \mathrm{keV}$ and a timing window of 6 ns. At least 1.5 million counts were acquired. Point source positions were ensured by examining reconstructed images using the FBP algorithm and adjusted manually until the center of the point source was located at the center of transaxial and axial FOVs. After acquiring data at the center of a FOV, the point source was moved in the vertical direction in steps of $1 \mathrm{~mm}$ from 0 to $10 \mathrm{~mm}$ and in steps of $2 \mathrm{~mm}$ from 12 to $32 \mathrm{~mm}$ (maximum offset) to acquire data for measuring off-center resolutions according to radial offset. The vertical direction in the gantry (real space) corresponded to the radial direction in transaxial images. Measurements along the radial direction were repeated 4 times with the source stepped along the axial direction using a step size of $0.2 \mathrm{~mm}$ (one eighth of the crystal pitch) to obtain oversampled axial profiles by interleaving (12).

List-mode PET data were sorted into 3D sinograms. Delayed and random events were subtracted from prompt events. 3D sinograms were rebinned into 2D sinograms using FORE and reconstructed using FBP with a ramp filter cutoff at the Nyquist frequency after detector normalization using the component-based normalization method (21). The pixel size of reconstructed images was $0.087 \times 0.087 \mathrm{~mm}(10 \times$ image zoom).

To determine transaxial radial and tangential resolutions at each position, profiles through count distribution peaks were drawn in 2 orthogonal directions on middle slices. FWHM and full width at tenth maximum (FWTM) values were then determined by linear interpolation between adjacent pixels at a half or tenth of the profile maximum value, which was determined by parabolic fit using peak points and their 2 nearest neighboring points (22,23). Axial profiles were also drawn to determine axial resolution with or

TABLE 1

Specification Comparison of microPET Systems Related to Focus 120

\begin{tabular}{|c|c|c|c|c|}
\hline Category & Specification & Focus 120 & $\mathrm{R} 4^{*}$ & Focus $220^{\dagger}$ \\
\hline \multirow[t]{5}{*}{ Detector } & Crystal material & LSO & LSO & LSO \\
\hline & Crystal element size $\left(\mathrm{mm}^{3}\right)$ & $1.5 \times 1.5 \times 10$ & $2.1 \times 2.1 \times 10$ & $1.5 \times 1.5 \times 10$ \\
\hline & Crystal pitch (mm) & 1.59 & 2.45 & 1.59 \\
\hline & Crystal array & $12 \times 12$ & $8 \times 8$ & $12 \times 12$ \\
\hline & Packing fraction (\%) & 92 & 80 & 92 \\
\hline \multirow[t]{8}{*}{ System } & Number of detector blocks & 96 & 96 & 168 \\
\hline & Total number of crystal elements & 13,824 & 6,144 & 24,192 \\
\hline & Number of rings & 48 & 36 & 48 \\
\hline & Ring diameter $(\mathrm{cm})$ & 15 & 15 & 26 \\
\hline & Bore size $(\mathrm{cm})$ & 12 & 12 & 22 \\
\hline & Transaxial FOV (cm) & 10 & 10 & 19 \\
\hline & Axial FOV $(\mathrm{cm})$ & 7.6 & 7.8 & 7.6 \\
\hline & Solid angle $/ 4 \pi$ & 0.45 & 0.46 & 0.28 \\
\hline
\end{tabular}

*Data published by Knoess et al. (19).

${ }^{\dagger}$ Data published by Tai et al. (12). 
without axial oversampling. Finally, volumetric resolution was calculated as the product of the axial, transaxial radial, and transaxial tangential resolutions.

\section{Sensitivity}

Sensitivity was defined by the ratio of the collected true coincidence counting rate to radioactivity (counts/s/kBq, cps/kBq). To measure sensitivity, a ${ }^{18} \mathrm{~F}$ line source $(148 \sim 370 \mathrm{kBq})$ was embedded in a glass capillary tube of the same length as the axial FOV of the scanner $(7.6 \mathrm{~cm})$; an inner tube diameter of $1.1 \mathrm{~mm}$ was used. The line source was placed along the axis of the scanner and centered in the transaxial and axial direction. After confirming that the dead-time loss was $<1 \%$, the line source, surrounded by $1 \sim 5$ aluminum sleeves of thickness $1 \mathrm{~mm}$ and different diameters, was scanned to determine the sensitivity free of photon attenuation and scatter. PET data were acquired for 10 min under 4 different conditions (energy window of $250 \sim 750$ or $350 \sim 650 \mathrm{keV}$; coincidence window of 6 or $10 \mathrm{~ns}$ ). The scan time was sufficiently long to collect $>10,000$ true counts. The number of coincidences was measured for every attenuating thickness(1, 2, 3, 4, and $5 \mathrm{~mm}$ ), and delayed coincidences and intrinsic radioactivities of LSO crystals (24-26) were subtracted to obtain true counting rates, which were corrected for ${ }^{18} \mathrm{~F}$ activity decay and plotted against attenuator thickness to extrapolate true counting rates with no attenuation from these measurements $(19,22,23,27)$. To assess sensitivity at the periphery of the transaxial FOV, the same experiments were repeated at a $20-\mathrm{mm}$ radial offset from the center. The measured sensitivity values using the line source were multiplied by 2 to obtain the absolute sensitivity values of the scanner for a central point source $(19,28)$ and corrected for the ${ }^{18} \mathrm{~F}$ branching ratio $(0.967)$.

In addition, the $3 \mathrm{D}$ sensitivity profile over the whole FOV was measured. The ${ }^{22} \mathrm{Na}$ point source $(673.4 \mathrm{kBq})$ was positioned at the center of the FOV, and the bed was moved in the axial and radial directions in steps of $4 \mathrm{~mm}$ using an energy window of $350 \sim 650 \mathrm{keV}$ and a coincidence window of $10 \mathrm{~ns}$.

\section{Scatter Fraction and Counting Rate Performance}

Scatter fraction and counting rate performance were measured using 2 different cylindric polyethylene "NEMA-like" phantoms (density, $0.96 \mathrm{~g} / \mathrm{mL}$ ), which simulated a typical rat body (inner diameter, $6 \mathrm{~cm}$; length, $15 \mathrm{~cm}$ ) and a mouse body (inner diameter, $3 \mathrm{~cm}$; length, $7 \mathrm{~cm}$ ). Holes were drilled parallel to the central axis of the cylinder at a radial distance of $15 \mathrm{~mm}$ (rat scatter phantom) and $7.5 \mathrm{~mm}$ (mouse scatter phantom). A line-source insert with the same length as each phantom was filled with ${ }^{18} \mathrm{~F}$ solution and threaded through these holes. Phantoms were mounted on the imaging bed and centered in transaxial and axial FOVs. Initial linesource activities were $335.748 \mathrm{MBq}(9.07 \mathrm{mCi})$ and $310.43 \mathrm{MBq}$ $(8.39 \mathrm{mCi})$ for mouse and rat phantoms, respectively. List-mode PET data were collected every $3 \mathrm{~min}$ for initial 6-h datasets and then for $10 \mathrm{~min}$ for later datasets. PET data were collected for a total of $23 \mathrm{~h}$ (12.5 half-lives) at all combinations of the 2 energy windows $(250 \sim 750$ and $350 \sim 650 \mathrm{keV})$ and 2 coincidence windows (6 and $10 \mathrm{~ns}$ ). PET data were acquired until dead-time loss was $<1 \%$ for scatter fraction calculations (14). Background coincidence events due to intrinsic true counts from ${ }^{176} \mathrm{Lu}$ in the LSO crystal was also measured with mouse or rat phantoms centered in the FOV with no activity in the line source (blank sinogram) $(24,26)$. PET list-mode data were sorted into 2D sinograms using SSRB. Prompt and random sinograms were generated separately. The random sinograms were estimated from singles event rates (13).
For prompt sinograms (bin size, $0.815 \mathrm{~mm}$; slice thickness, $0.796 \mathrm{~mm}$ ) of each slice, the projection profile of each projection angle was shifted so that peak pixels were aligned with the center of the sinogram and the summed projection profile across the projection angle was then generated. For each summed profile, all counts beyond a band of $16 \mathrm{~mm}$ larger than the phantom were set to zero. All pixel counts beyond a 7-mm radius (line-source band) from the center of profile were assumed to be the sum of random, scatter, and intrinsic counts, and these counts under the peak were estimated by linearly interpolating the number of events at the left and right borders of the line-source band. Counts above this line were regarded as true counts. Random and intrinsic counts were estimated from random and blank sinograms, respectively, using the same band size of $16 \mathrm{~mm}$ larger than the phantom $(13,26)$.

The scatter counting rate was calculated using the following equation:

$$
R_{\text {scatter }}=R_{\text {total }}-R_{\text {true }}-R_{\text {random }}-R_{\text {intrinsic }},
$$

where $R_{\text {scatter }}, R_{\text {total }}, R_{\text {true }}, R_{\text {random }}$, and $R_{\text {intrinsic }}$ are the scatter, total, true, random, and intrinsic counting rates, respectively. Scatter fractions for slices were calculated as the ratios of scattered and total events and averaged to obtain overall system scatter fractions.

Noise equivalent counting rate (NECR) at the $i$ th acquisition was then determined using the following equation:

$$
\frac{R_{\text {trues }}(i)^{2}}{R_{\text {total }}(i)},
$$

Eq. 2

where $R_{\text {total }}(i)$ and $R_{\text {true }}(i)$ are the total and true counting rates, respectively, for the $i$ th acquisition $(12,13,23,26)$.

\section{Imaging Studies}

Phantom Study. A Micro Deluxe phantom (inner diameter, 4.5 $\mathrm{cm}$; inside height, $6.3 \mathrm{~cm}$ ) with different sized hot-rod inserts (diameters, 1.2 1.6, 2.4, 3.2, 4.0, and $4.8 \mathrm{~mm}$ ) arranged in 6 segments (Data Spectrum Corp.) was scanned with an energy window of $350 \sim 650 \mathrm{keV}$ and a coincidence window of $6 \mathrm{~ns}$. The phantom was filled with $9.3 \mathrm{MBq}(250 \mu \mathrm{Ci})$ of ${ }^{18} \mathrm{~F}$ and scanned for $1 \mathrm{~h}$. The PET image was reconstructed using 3DRP (16).

Animal Studies. To investigate the feasibility of the scanner for animal studies, mouse, rat, and cat studies were performed.

A mouse bone image was acquired using the same energy and coincidence windows as used in the phantom study. ${ }^{18} \mathrm{~F}(41.8 \mathrm{MBq}$ [1.13 mCi]) was injected intravenously and, $3 \mathrm{~h}$ later, a 1-h list-mode emission dataset was acquired. List-mode PET data were sorted into sinograms and reconstructed using different reconstruction algorithms including FBP, 3DRP, expectation maximinization (EM), and MAP (iterations, $40 ; \beta, 0.0001$ ).

A cardiac gated PET image of a 200-g Sprague-Dawley rat was acquired. Thirty-seven megabecquerels $(1 \mathrm{mCi})$ of ${ }^{18} \mathrm{~F}-\mathrm{FDG}$ were injected intravenously and, $1 \mathrm{~h}$ later, a 30-min list-mode emission dataset was acquired. Sinograms were rebinned using FORE and reconstructed using the OSEM algorithm.

An ${ }^{18}$ F-FDG scan of a cat brain was also acquired. A $2.5-\mathrm{kg}$ cat was positioned in the scanner with its brain centered in the FOV. A 30 -min transmission scan was acquired using a ${ }^{68} \mathrm{Ge}$ point source with an energy window of $460 \sim 560 \mathrm{keV}$ and singles mode. ${ }^{18} \mathrm{~F}$ FDG (92.5 MBq [2.5 mCi]) was injected intravenously and, $30 \mathrm{~min}$ 
later, 30 min of list mode of the emission dataset was acquired. An attenuation map was reconstructed from the transmission PET data and a blank scan using MAP reconstructed transmission images (MAP TR) (29) and reprojected to the sinogram space using inverse FORE to form an attenuation correction file.

\section{RESULTS}

\section{Energy Resolution}

The best energy resolution for all crystals was $14.4 \%$, and the worst energy resolution was $41.4 \%$. The energy resolution at $511 \mathrm{keV}$, averaged over all heads, detectors, and crystals, was $18.3 \%$, which was better than that of the R4 system (23\%) (19) and was equivalent to that of the F220 system $(18.5 \%)(12)$. Thus, energy resolution was found to be significantly improved on the F120 system as compared with the R4 system.

\section{Spatial Resolution}

Radial, tangential, and axial spatial resolutions acquired from the FBP reconstructed images of ${ }^{22} \mathrm{Na}$ point source are plotted versus the radial offset from the center (Figs. 1A and 1B). Resolutions using FBP were 1.18 (radial), 1.13 (tangential), 1.44 (axial without oversampling), and 1.45 mm FWHM (axial with oversampling) at the center and 2.35 (radial), 1.66 (tangential), 2.09 (axial without oversampling), and $2.00 \mathrm{~mm}$ FWHM (axial with oversampling) at a radial offset of $2 \mathrm{~cm}$. Volumetric resolution is shown in Figure 1C. The volumetric resolution was $1.92 \mu \mathrm{L}$ without axial oversampling and $1.94 \mu \mathrm{L}$ with axial oversampling at the center, and $8.14 \mu \mathrm{L}$ without axial oversampling and $7.81 \mu \mathrm{L}$ with axial oversampling at a radial offset of $2 \mathrm{~cm}$.

\section{Sensitivity}

At the center of the axial FOV, the absolute sensitivity at the transaxial center and with a $20-\mathrm{mm}$ radial offset using an energy window of $250 \sim 750 \mathrm{keV}$ and a coincidence window of $10 \mathrm{~ns}$ were 69.9 and $71.2 \mathrm{cps} / \mathrm{kBq}$ (7.0 and $7.1 \%$ ), respectively. Absolute sensitivity values at the center and with a $20 \mathrm{~mm}$ radial offset with other combinations of energy and coincidence windows are summarized in Table 2. Figure 2 shows the sensitivity profile measured using a ${ }^{22} \mathrm{Na}$ point source as a function of transaxial and axial positions with an energy window of $350 \sim 650$ and a coincidence window of $10 \mathrm{~ns}$.

\section{Scatter Fraction and Counting Rate Performance}

The scatter fraction and NECR for mouse and rat phantoms are summarized in Table 3. With an energy window of $250 \sim 750 \mathrm{keV}$ and a coincidence window of $6 \mathrm{~ns}$, the peak true counting rates for mouse and rat phantoms were 1,296 kcps at $3,243 \mathrm{kBq} / \mathrm{mL}$ and $582 \mathrm{kcps}$ at $448 \mathrm{kBq} / \mathrm{mL}$, respectively. The NECR curves for the mouse and rat phantoms are plotted as a function of total activity in the phantom in Figures 3A and 3B. With an energy window of $250 \sim 750 \mathrm{keV}$ and a coincidence window of $6 \mathrm{~ns}$, the peak NECRs for mouse and rat phantoms were $869 \mathrm{kcps}$ at 3,242
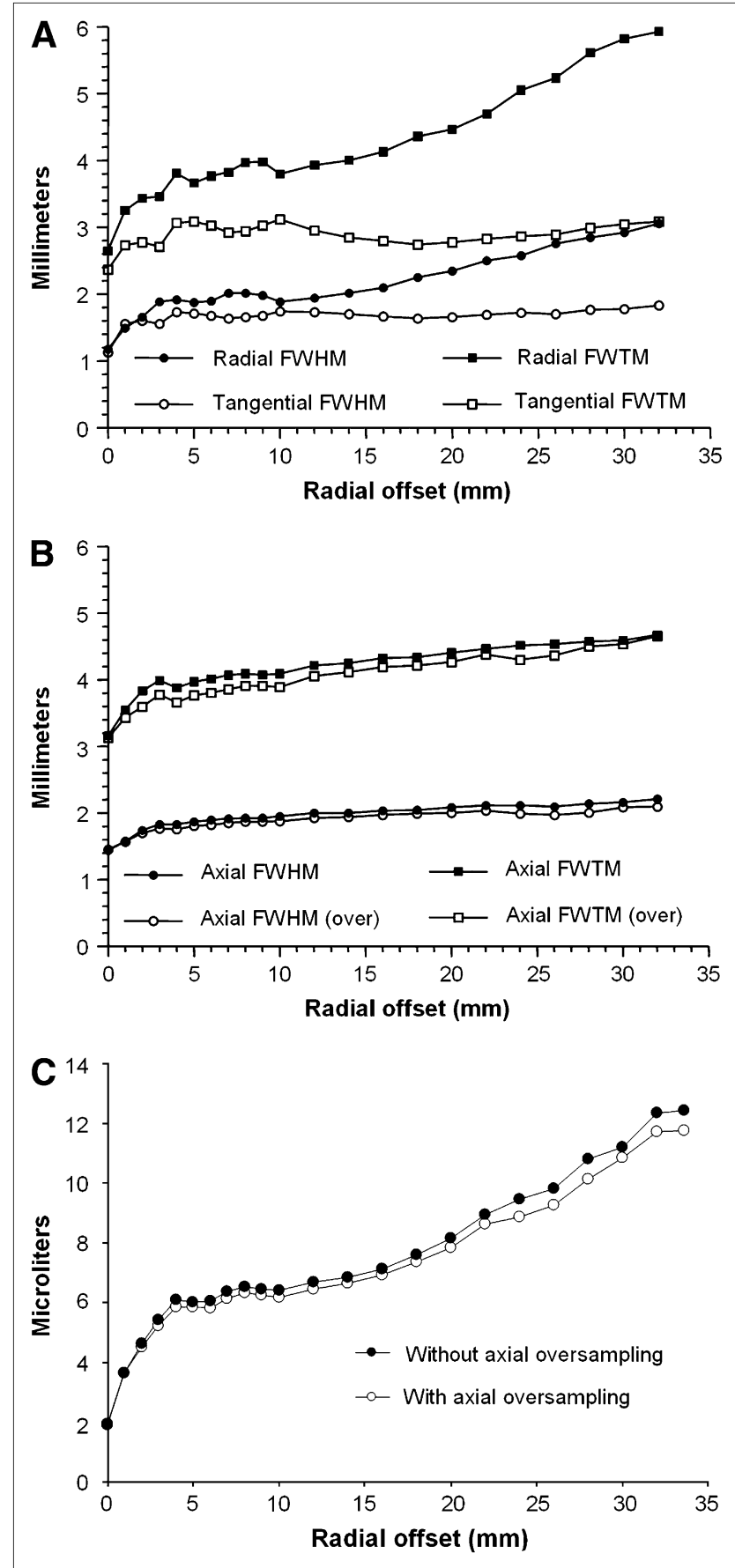

FIGURE 1. Spatial resolution profile measured using a ${ }^{22} \mathrm{Na}$ point source. (A) Transaxial (radial and tangential). (B) Axial with or without oversampling. (C) Volume resolution with or without oversampling.

$\mathrm{kBq} / \mathrm{mL}$ ( $4.34 \mathrm{mCi}$ total activity) and $228 \mathrm{kcps}$ at $290 \mathrm{kBq} / \mathrm{mL}$ (3.32 mCi total activity), respectively.

\section{Imaging Studies}

Phantom Study. Figure 4 shows a transaxial image of the Micro Deluxe phantom with hotrod inserts. All rod inserts with diameters of $1.6 \sim 4.8 \mathrm{~mm}$ were distinguishable in this 
TABLE 2

Absolute Sensitivity for a Point Source Centered in Axial FOV

\begin{tabular}{lccc}
\hline $\begin{array}{c}\text { Radial } \\
\text { distance }(\mathrm{mm})\end{array}$ & $\begin{array}{c}\text { Energy } \\
\text { window }(\mathrm{keV})\end{array}$ & $\begin{array}{c}\text { Coincidence } \\
\text { window }(\mathrm{ns})\end{array}$ & $\begin{array}{c}\text { Sensitivity } \\
(\%)\end{array}$ \\
\hline 0 & $250-750$ & 10 & 7.0 \\
& & 6 & 6.7 \\
& $350-650$ & 10 & 4.0 \\
20 & & 6 & 3.8 \\
& $250-750$ & 10 & 7.1 \\
& & 6 & 6.9 \\
& $350-650$ & 10 & 3.0 \\
& & 6 & 2.9 \\
\hline
\end{tabular}

image. Although the rods with diameter of $1.2 \mathrm{~mm}$ were identifiable, their separation was not clear.

Animal Studies. Figure 5 shows maximum-intensity projection (MIP) images of the ${ }^{18} \mathrm{~F}$ PET mouse bone scan. Bony structures in mouse, including spine, rib cages, and skull, are clearly visualized.

Figure 6A shows the heart images of a normal rat acquired $20 \mathrm{~min}$ after ${ }^{18} \mathrm{~F}-\mathrm{FDG}$ injection. Uniform ${ }^{18} \mathrm{~F}-$ FDG uptake throughout the myocardium of the left ventricle was clearly resolved.

Figure 6B shows a cat brain ${ }^{18} \mathrm{~F}-\mathrm{FDG}$ PET image and demonstrates the feasibility of this scanner for cat brain imaging. The whole head of the cat is included within the transaxial FOV and the brain cortex was no more than $2 \mathrm{~cm}$ from the center of the FOV. Figure $6 \mathrm{~B}$ also demonstrates the substantially improved spatial resolution of this scanner (Fig. 14 in (19)), as the gyrus convolutions in the cat brain are well visualized.

\section{DISCUSSION}

In this study, we evaluated the performance characteristics of the F120 PET scanner. Results of our evaluation are

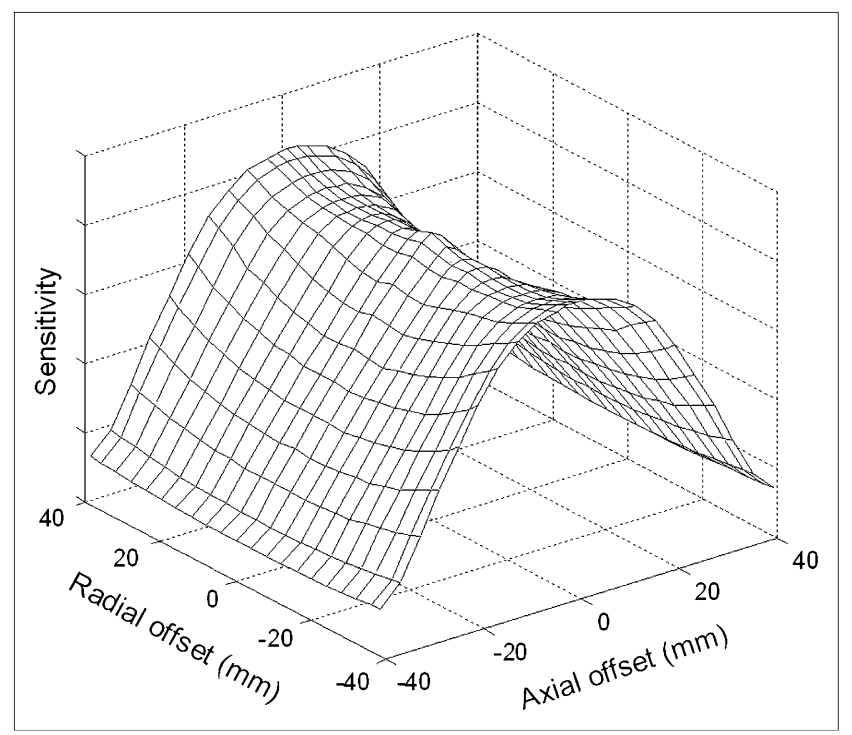

FIGURE 2. 3D sensitivity profile over a FOV. summarized in Table 4 and compared with those of related previously published microPET series systems (i.e., R4 and F220) $(12,19)$. The R4 is the forerunner of the F120; they have the same ring diameters and axial lengths but different crystal sizes (R4, $2.1 \mathrm{~mm}$; Focus, $1.5 \mathrm{~mm}$ ). The F220 has essentially the same detector and electronics as the F120 but a larger ring diameter $(26 \mathrm{~cm}$ vs. $15 \mathrm{~cm})$. The evaluation results (13) for the F120 produced by another group are also compared.

The average energy resolution was better for the F120 as compared with the R4 scanner (23\%-18\%) (19), which was equivalent to the result obtained using the F220 (12). Our results also show that the spatial resolution of the F120 has been remarkably improved $(1.18 \mathrm{~mm}$ in the radial, $1.13 \mathrm{~mm}$ in the tangential, and $1.44 \mathrm{~mm}$ in the axial directions) as compared with the R4 $(1.65,1.66$, and $1.84 \mathrm{~mm}$, respectively) at the FOV centers (19). These results were obtained from ${ }^{22} \mathrm{Na}$ point-source images reconstructed using FORE and 2D FBP. However, direct comparisons between these values are not appropriate because point sources with different diameters were used for the resolution measurements (1.0 $\mathrm{mm}$ in (19) and $0.25 \mathrm{~mm}$ in our study). After correcting for source dimension, differences between these values were diminished but the spatial resolution of the F120 $(1.15,1.10$, and $1.42 \mathrm{~mm})$ remained better than that of the $\mathrm{R} 4(1.31,1.33$, and $1.54 \mathrm{~mm})$. Radial and tangential resolution values corrected for source dimension using the relationship below $(30,31)$ for both scanners approximately matched predicted values $(1.16 \mathrm{~mm}$ for F120 and $1.48 \mathrm{~mm}$ for R4):

$F W H M=1.25 \sqrt{\left(\frac{D_{\text {crystal }}}{2}\right)^{2}+\left(0.0022 D_{\text {system }}\right)^{2}+p^{2}+b^{2}}$,

Eq. 3

where, $D_{\text {crystal }}$ is the dimension of crystal elements, $D_{\text {system }}$ is the ring diameter, $p$ is the positron range $(0.45 \mathrm{~mm}$ for ${ }^{22} \mathrm{Na}$ ) (32), and $b^{2}$ is the plausible nonzero block factor (assumed to be zero).

In PET scanners of a small ring diameter and crystal size, such as the F120, radial spatial resolution in the peripheral region of the transaxial FOV is poorer than that at the FOV center. This is due to parallax errors caused when the photons impinge on the detectors at oblique angles (9). In this study, such degradations in radial resolution (parallax error) at the periphery of FOVs were observed $(1.18 \mathrm{~mm}$ at the center vs. $2.35 \mathrm{~mm}$ at $2 \mathrm{~cm}$ off-center). However, this radial resolution at $2 \mathrm{~cm}$ off-center is similar to that of the GE eXplore VISTA $(\sim 2 \mathrm{~mm})$, which provides a 2-valued estimate of the depth at which an annihilation photon is absorbed by the phoswich crystal elements to avoid this problem The phoswich element consists of 2 different crystals (lutetium-yttrium oxyorthosilicate [LYSO] and germanium oxyorthosilicate [GSO)]), and the depth of interaction (DOI) is identified by measuring the decay time 
TABLE 3

Scatter Fraction and Counting Rate Performance

\begin{tabular}{|c|c|c|c|c|c|}
\hline Phantom & $\begin{array}{c}\text { Energy } \\
\text { window (keV) }\end{array}$ & $\begin{array}{l}\text { Coincidence } \\
\text { window (ns) }\end{array}$ & $\begin{array}{c}\text { Scatter } \\
\text { fraction (\%) }\end{array}$ & $\begin{array}{c}\text { Peak true } \\
\text { (kcps at } \mathrm{kBq} / \mathrm{mL})\end{array}$ & $\begin{array}{c}\text { Peak NECR } \\
\text { (kcps at } \mathrm{kBq} / \mathrm{mL})\end{array}$ \\
\hline \multirow[t]{4}{*}{ Mouse } & $250 \sim 750$ & 6 & 15.9 & 1,296 at 3,242 & 869 at 3,242 \\
\hline & & 10 & 15.0 & 1,218 at 2,136 & 793 at 2,136 \\
\hline & $350 \sim 650$ & 6 & 11.6 & 1,088 at 3,684 & 741 at 2,989 \\
\hline & & 10 & 11.0 & 1,092 at 3,108 & 689 at 2,781 \\
\hline \multirow[t]{4}{*}{ Rat } & $250 \sim 750$ & 6 & 35.0 & 582 at 448 & 228 at 290 \\
\hline & & 10 & 34.9 & 585 at 358 & 199 at 262 \\
\hline & $350 \sim 650$ & 6 & 22.9 & 449 at 506 & 201 at 289 \\
\hline & & 10 & 23.1 & 466 at 498 & 175 at 260 \\
\hline
\end{tabular}

(LYSO, $40 \mathrm{~ns}$; GSO, $60 \mathrm{~ns}$ ) of the light pulse of each event. The DOI information allows the reduction of uncertainty in the interaction position and, as a consequence, the effects of parallax error. (33). Volume resolutions at center position were also similar for the 2 units $(7.81 \mu \mathrm{L}$ in F120 and 7.0 $\mu \mathrm{L}$ in VISTA). The images of cylindric phantoms with hotrod inserts shown in Figure 4, and of cat brain shown in Figure 6B, also demonstrate that the spatial resolution is fairly homogeneous within a FOV of $<2 \mathrm{~cm}$.

Sensitivity of the PET system is determined by several factors, which include solid angle, packing fraction, and scintillator efficiency for $511-\mathrm{keV}$ photons. The sensitivity of the F120 unit was 2-fold that of the F220, although the solid angle of the F120 is only $60 \%$ larger than that of the

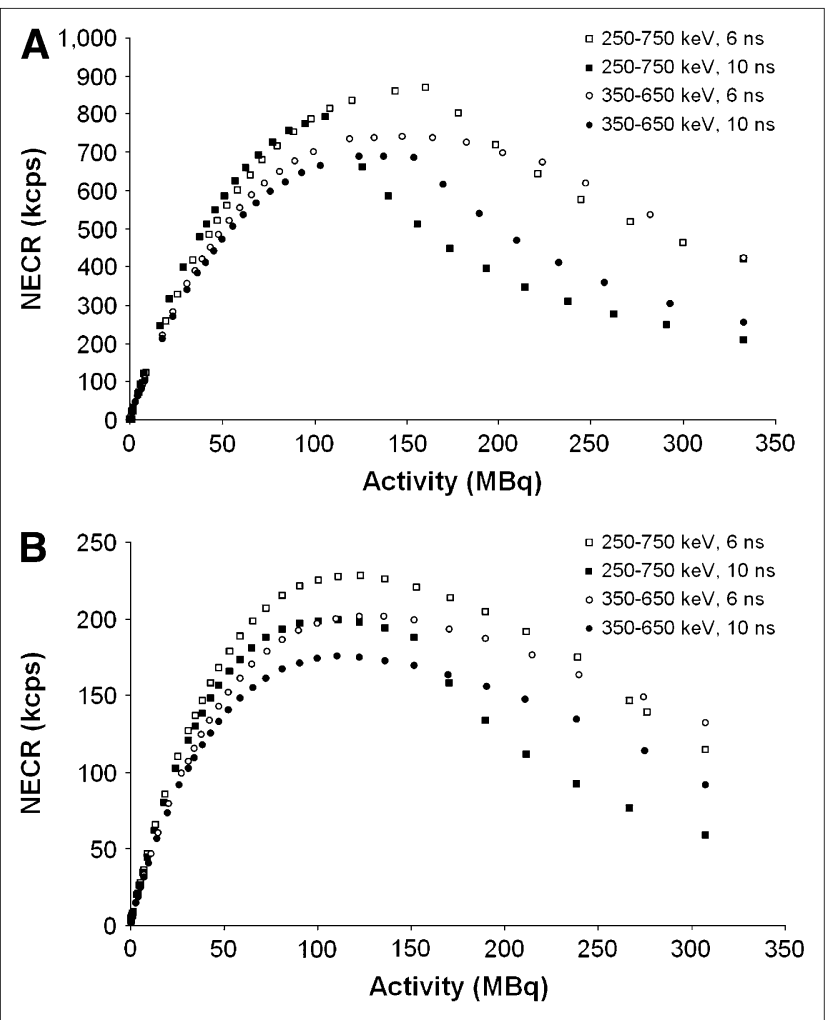

FIGURE 3. NECR vs. total radioactivity for mouse (A) and rat (B) phantoms.
F220. A possible source of this discrepancy is the different methods used to measure the sensitivity. Although we measured the absolute sensitivity free of photon attenuation and scatter using an extrapolation method (27), the sensitivity of the F220 in (12) was measured using a point source encapsulated in a stainless steel sleeve. It is likely that their sensitivity was underestimated because the scattered photons in the source housing were lost during the PET scan. The observed improvement in sensitivity of the F120 over the R4 was $55 \%$.

The counting rate performance of a PET scanner is a function of several scanning parameters. In this study, we investigated counting rate performance in terms of the scatter fraction and the NECR in a wide range of situations to optimize the scan protocol. For both mouse and rat phantoms, peak NECR values were obtained with an energy window of $250 \sim 750 \mathrm{keV}$ and a coincidence window of $6 \mathrm{~ns}$, although scatter fractions under these conditions were higher than those obtained with a narrower energy window.

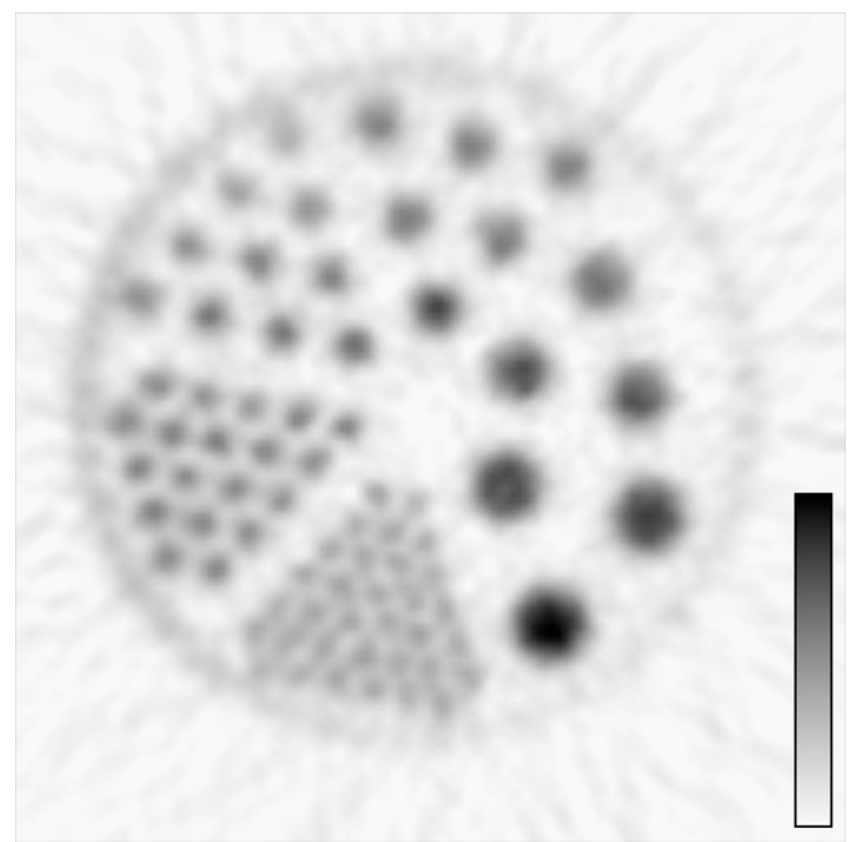

FIGURE 4. Image of Micro Deluxe phantom with hot-rod inserts - reconstructed using the 3DRP algorithm. 

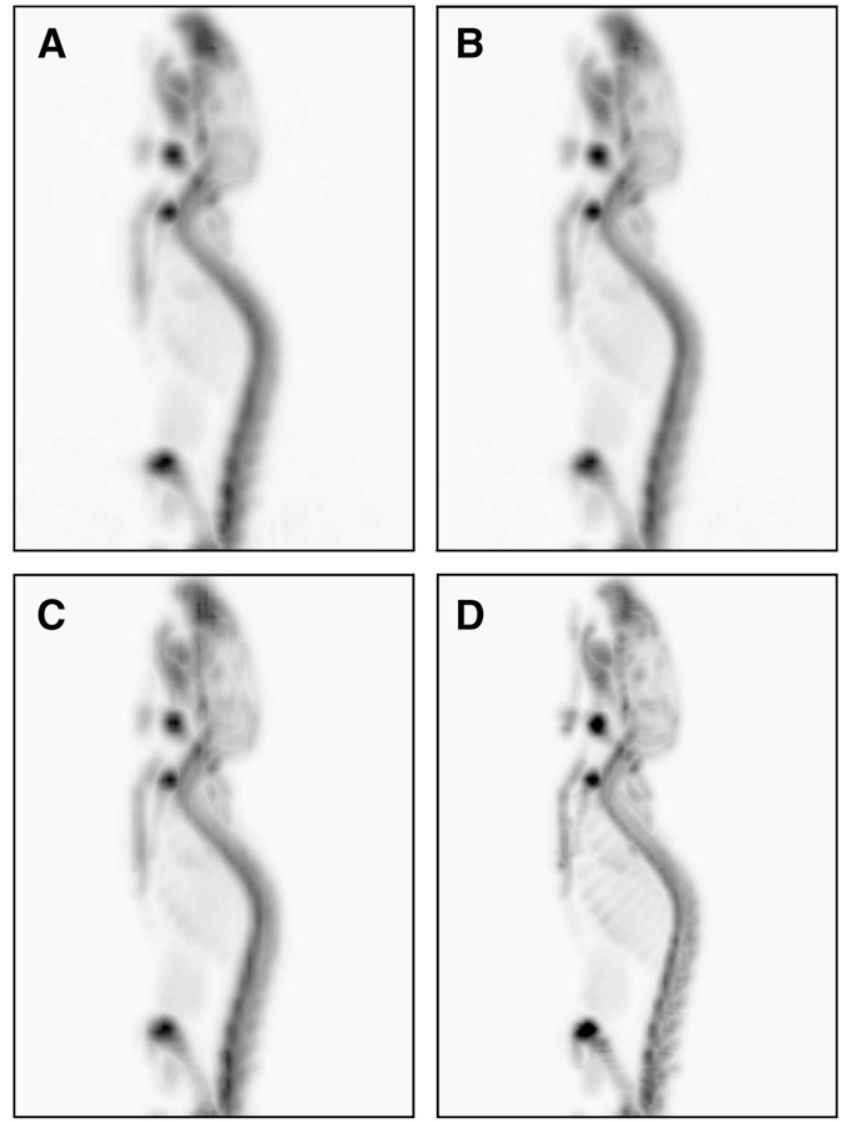

FIGURE 5. Maximum-intensity projection (MIP) images of mouse bone ${ }^{18} \mathrm{~F}$ PET reconstructed using different algorithms: (A) FBP, (B) 3DRP, (C) EM, and (D) MAP.

These scanning parameters are suitable for the F120 scanner in terms of counting rate performance. However, only qualitative comparisons are possible because different sized phantoms were used to obtain NECR measurements. The peak NECR of the F120 scanner was also much better than that of the R4 scanner (19) and noticeably higher than that of the F220 (12) ( 30\% improvement) (Table 4). It seems, however, that the higher peak NECR for our mouse phan- tom compared with the R4 data is mainly due to the different diameters of the phantoms used for NECR measurements ( $3 \mathrm{~cm}$ in our work and $4.3 \mathrm{~cm}$ in (19)). When the same mousesize phantom was scanned on the F120 and R4, similar peak NECR values were obtained (13). They explained that this was because the counting rate performance is limited by the data throughput at the host personal computer level.

Compared with the previous study by Laforest et al. (13), we measured spatial resolution at a smaller radial offset interval from FOV centers (i.e., in steps of $1 \mathrm{~mm}$ from 0 to $10 \mathrm{~mm}$, and in steps of $2 \mathrm{~mm}$ from 12 to $32 \mathrm{~mm}$ ) and used a smaller point source. Resolution was measured in the previous study at only 4 radial offsets. To more accurately determine the axial resolution at each radial position, we repeated each point-source measurement 4 times using different axial offsets. In addition, we measured the 3D sensitivity profile over the whole FOV. The detailed information obtained will be useful for predicting spatially varying partial-volume effects and for improving the spatial resolution of reconstructed PET images.

The resolution and sensitivity values measured in the present study were similar to those measured in the previous study (Table 4). However, scatter fraction and peak NECR differ because cylindric phantoms with different diameters were used. We used the phantoms with slightly larger diameters and obtained higher scatter fractions for both phantoms. For the rat phantom, a lower peak NECR was observed with a lower activity level. However, the peak NECR for mouse phantom was higher in our study, which is not concordant with the general relationship between the NECR peak and phantom size. There is also a discrepancy in the activity level at the NECR peak $(4.34 \mathrm{mCi}$ for our work and $2.4 \mathrm{mCi}$ for Laforest et al.). Slow change in the NECR curves around the peak (especially in Laforest et al.) could be the reason for this difference in activity. Nevertheless, general trends were similar; the rat phantom showed a higher scatter fraction and a lower peak NECR, and the peak NECR was obtained with an energy window of $250 \sim 750 \mathrm{keV}$ and a coincidence window of $6 \mathrm{~ns}$ for both mouse and rat phantoms.

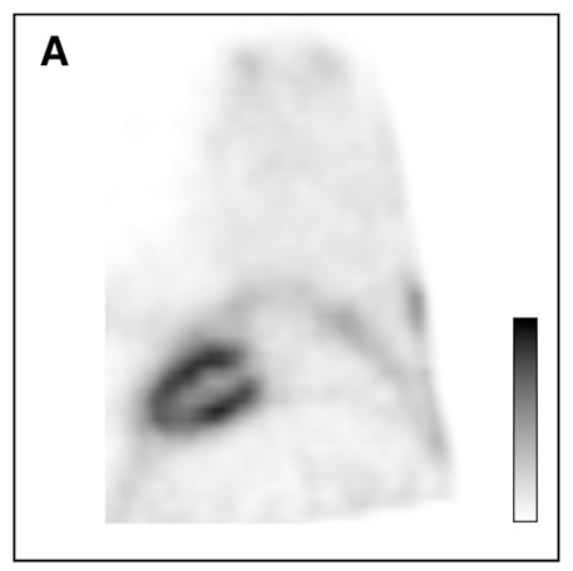

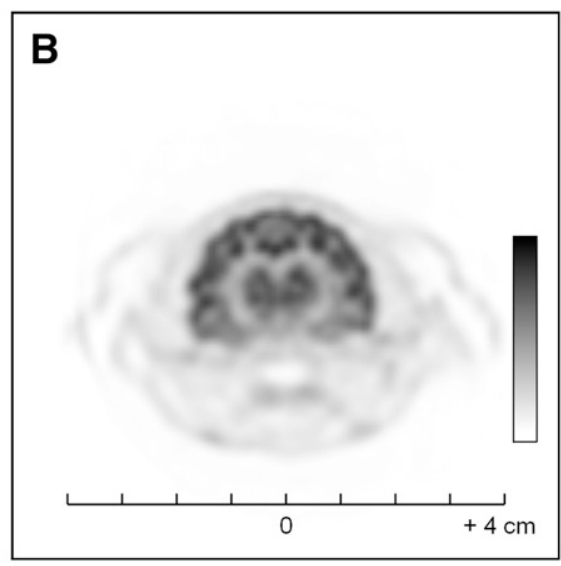

FIGURE 6. Selected animal imaging studies. (A) Myocardial PET image of rat in end-diastolic phase. (B) Cat brain PET image acquired using ${ }^{18} \mathrm{~F}-\mathrm{FDG}$ and reconstructed using the MAP algorithm. 
TABLE 4

Comparison of Performance Characteristics in microPET Scanners

\begin{tabular}{|c|c|c|c|c|c|}
\hline \multicolumn{2}{|c|}{ Performance characteristic } & \multirow{2}{*}{$\begin{array}{c}\begin{array}{c}\text { Focus } 120 \\
\text { (this study) }\end{array} \\
18.3\end{array}$} & \multirow{2}{*}{$\begin{array}{c}\begin{array}{c}\text { Focus } 120 \\
(\text { Laforest et al.) }\end{array} \\
\text { N/A }\end{array}$} & \multirow{2}{*}{$\frac{\mathrm{R} 4^{\dagger}}{23}$} & \multirow{2}{*}{$\frac{\text { Focus } 220^{\ddagger}}{18.5}$} \\
\hline Energy resolution (\%) & & & & & \\
\hline \multirow{3}{*}{$\begin{array}{l}\text { Volumetric resolution } \\
(\mu L)^{\S}\end{array}$} & Center & 1.92 & 2.87 & 5.1 & 2.5 \\
\hline & $1-\mathrm{cm}$ radial offset & 6.41 & $\mathrm{~N} / \mathrm{A}$ & 15.6 & $\mathrm{~N} / \mathrm{A}$ \\
\hline & $2-\mathrm{cm}$ radial offset & 8.14 & 8.23 & $\sim 19 \|$ & 6.3 I \\
\hline \multirow{4}{*}{$\begin{array}{l}\text { Absolute sensitivity for } \\
\text { point source at } \\
\text { center }(\%)\end{array}$} & $250 \sim 750 \mathrm{keV}, 10 \mathrm{~ns}$ & 7.0 & 7.1 & N/A & 3.4 \\
\hline & $250 \sim 750 \mathrm{keV}, 6 \mathrm{~ns}$ & 6.7 & $6.7^{\star \star}$ & 4.37 & 3.0 \\
\hline & $350 \sim 650 \mathrm{keV}, 10 \mathrm{~ns}$ & 4.0 & $4.7^{\star \star}$ & $\mathrm{N} / \mathrm{A}$ & $\mathrm{N} / \mathrm{A}$ \\
\hline & $350 \sim 650 \mathrm{keV}, 6 \mathrm{~ns}$ & 3.8 & $4.4^{\star \star}$ & 2.45 & 2.1 \\
\hline \multirow[t]{2}{*}{ Scatter fraction $(\%)^{\dagger \dagger}$} & Mouse phantom & $\begin{array}{c}15.9 \\
(3-\mathrm{cm} \varnothing .7-\mathrm{cm} \mathrm{L})\end{array}$ & $\begin{array}{c}12.3 \\
(2.5-\mathrm{cm} \varnothing .7-\mathrm{cm} \mathrm{L})\end{array}$ & $\begin{array}{c}32.0 \\
(4.3-\mathrm{cm} \varnothing .7 .6-\mathrm{cm} \mathrm{L})\end{array}$ & $\begin{array}{c}19.0 \\
(3-\mathrm{cm} \varnothing .7-\mathrm{cm} \mathrm{L})\end{array}$ \\
\hline & Rat phantom & $\begin{array}{c}35.0 \\
(6-\mathrm{cm} \varnothing, 15-\mathrm{cm} \mathrm{L})\end{array}$ & $\begin{array}{c}26.3 \\
(5-\mathrm{cm} \varnothing, 15-\mathrm{cm} \mathrm{L})\end{array}$ & $\begin{array}{c}38.0 \\
(6-\mathrm{cm} \varnothing, 9.6-\mathrm{cm} \mathrm{L})\end{array}$ & $\begin{array}{c}36.4 \\
(6-\mathrm{cm} \varnothing, 15-\mathrm{cm} \mathrm{L})\end{array}$ \\
\hline \multirow[t]{3}{*}{ Peak NECR $(\mathrm{kcps})^{\dagger \dagger}$} & Mouse phantom & 869 at $160.58 \mathrm{MBq}$ & $\begin{array}{l}809 \text { at } 88.8 \mathrm{MBq} \\
(2.4 \mathrm{mCi})\end{array}$ & $\begin{array}{l}174 \text { at } 76.96 \mathrm{MBq} \\
(2.08 \mathrm{mCi})\end{array}$ & $\begin{array}{l}645 \text { at } 146.52 \mathrm{MBq} \\
(3.96 \mathrm{mCi})\end{array}$ \\
\hline & & $(3-\mathrm{cm} \varnothing, 7-\mathrm{cm} \mathrm{L})$ & $(2.5-\mathrm{cm} \varnothing, 7-\mathrm{cm} \mathrm{L})$ & (4.3-cm Ø, 7.6-cm L) & (3-cm Ø, 7-cm L) \\
\hline & Rat phantom & $\begin{array}{l}228 \text { at } 122.84 \mathrm{MBq} \\
(3.32 \mathrm{mCi}) \\
(6-\mathrm{cm} \varnothing, 15-\mathrm{cm} \mathrm{L})\end{array}$ & $\begin{array}{l}300 \text { at } 149.85 \mathrm{MBq} \\
(4.05 \mathrm{mCi}) \\
(5-\mathrm{cm} \varnothing, 15-\mathrm{cm} \mathrm{L})\end{array}$ & $\begin{array}{l}94 \text { at } 59.94 \mathrm{MBq} \\
(1.62 \mathrm{mCi}) \\
(6-\mathrm{cm} \varnothing, 9.6-\mathrm{cm} \mathrm{L})\end{array}$ & $\begin{array}{l}177 \text { at } 143.19 \mathrm{MBq} \\
(3.87 \mathrm{mCi}) \\
(6-\mathrm{cm} \varnothing, 15-\mathrm{cm} \mathrm{L})\end{array}$ \\
\hline
\end{tabular}

*Data published by Laforest et al. (13).

†Data published by Knoess et al. (19).

${ }^{\ddagger}$ Data published by Tai et al. (12).

$\S_{\text {Data }}$ were not corrected for source dimension, positron range, or acolinearity of positron annihilation and were estimated from nonoversampled axial profiles except for Focus 220.

"Axial resolution was estimated from oversampled axial profiles (Tai et al.) (12).

"Estimated from Figure 5 in Knoess et al. (19).

${ }^{\star}$ Estimated from Figure 1 in Laforest et al. (13).

${ }^{\dagger \dagger}$ Data were measured using same energy window (250-750 keV) and coincidence window (6 ns) but the phantom sizes ( $\varnothing$, diameter; L, length) were not identical.

$\mathrm{N} / \mathrm{A}=$ not available.

\section{CONCLUSION}

The results of this independent field test showed the improved physical characteristics of the F120 scanner over the previous microPET series systems. Compared with the R4 system, the energy resolution for the 511-keV photon and the volume resolution at the center were improved from $23 \%$ to $18.3 \%$ and from $5.1 \mu \mathrm{L}$ to $1.92 \mu \mathrm{L}$, respectively. The observed improvement in sensitivity of the F120 over the R4 was 55\%. The peak NECR of the F120 scanner was also much better than that of the R4 scanner. This system will be useful for imaging studies on the small rodents and the brains of larger animals.

\section{ACKNOWLEDGMENTS}

This work was supported by the Real Time Molecular Imaging Research Program and the Basic Research Program (grant R01-2006-000-10296-0) of the Korean Science \& Engineering Foundation funded by the Ministry of Science and Technology, Korea. The authors appreciate the experimental support of Sang Hee Han.

\section{REFERENCES}

1. Bateman TM. Cardiac positron emission tomography and the role of adenosine pharmacologic stress. Am J Cardiol. 2004;94:19D-24D.
2. Been LB, Suurmeijer AJ, Cobben DC, Jager PL, Hoekstra HJ, Elsinga PH. ${ }^{18} \mathrm{~F}-$ FLT-PET in oncology: current status and opportunities. Eur J Nucl Med Mol Imaging. 2004;31:1659-1672.

3. Nordberg A. PET imaging of amyloid in Alzheimer's disease. Lancet Neurol. 2004;3:519-527.

4. Rohren EM, Turkington TG, Coleman RE. Clinical applications of PET in oncology. Radiology. 2004;231:305-332.

5. Phelps ME. Nuclear medicine, molecular imaging, and molecular medicine. J Nucl Med. 2002;43:13N-14N.

6. Pomper MG. Can small animal imaging accelerate drug development? J Cell Biochem Suppl. 2002;39:211-220.

7. Gambhir SS. Molecular imaging of cancer with positron emission tomography. Nat Rev Cancer. 2002;2:683-693.

8. Pomper MG, Lee JS. Small animal imaging in drug development. Curr Pharm Des. 2005;11:3247-3272.

9. Green MV, Seidel J, Vaquero JJ, Jagoda E, Lee I, Eckelman WC. High resolution PET, SPECT and projection imaging in small animals. Comput Med Imaging Graph. 2001;25:79-86.

10. Chatziioannou AF. PET scanners dedicated to molecular imaging of small animal models. Mol Imaging Biol. 2002;4:47-63.

11. Hume SP, Myers R. Dedicated small animal scanners: a new tool for drug development? Curr Pharm Des. 2002;8:1497-1511.

12. Tai YC, Ruangma A, Rowland D, et al. Performance evaluation of the microPET focus: a third-generation microPET scanner dedicated to animal imaging. $J$ Nucl Med. 2005;46:455-463.

13. Laforest R, Longford D, Siegel S, Newport DF, Yap J. Performance evaluation of the microPET-Focus-F120. Conference Records IEEE Nuclear Science Symposium and Medical Imaging, Rome, Italy, October 16-22, 2004. Piscataway, NJ: IEEE; 2004.

14. Daube-Witherspoon ME, Muehllehner G. Treatment of axial data in threedimensional PET. J Nucl Med. 1987;28:1717-1724.

15. Yao R, Seidel J, Johnson CA, Daube-Witherspoon ME, Green MV, Carson RE. Performance characteristics of the 3-D OSEM algorithm in the reconstruction of 
small animal PET images: ordered-subsets expectation-maximixation. IEEE Trans Med Imaging. 2000;19:798-804.

16. Kinahan PE, Rogers JG. Analytic 3D image reconstruction using all detected events. IEEE Trans Nucl Sci. 1989;36:964-968.

17. Qi J, Leahy RM, Cherry SR, Chatziioannou A, Farquhar TH. High-resolution 3D bayesian image reconstruction using the microPET small-animal scanner. Phys Med Biol. 1998;43:1001-1013.

18. Defrise M, Kinahan PE, Townsend DW, Michel C, Sibomana M, Newport DF. Exact and approximate rebinning algorithms for 3-D PET data. IEEE Trans Med Imaging. 1997;16:145-158.

19. Knoess C, Siegel S, Smith A, et al. Performance evaluation of the microPET R4 PET scanner for rodents. Eur J Nucl Med Mol Imaging. 2003;30:737-747.

20. Weber S, Bauer A. Small animal PET: aspects of performance assessment. Eur J Nucl Med Mol Imaging. 2004;31:1545-1555.

21. Badawi RD, Marsden PK. Developments in component-based normalization for 3D PET. Phys Med Biol. 1999;44:571-594.

22. Daube-Witherspoon ME, Karp JS, Casey ME, et al. PET performance measurements using the NEMA NU 2-2001 standard. J Nucl Med. 2002;43:1398-1409.

23. National Electrical Manufacturers Association. NEMA Standards Publication NU2-2001: Performance Measurements of Positron Emission Tomographs. Rosslyn, VA: National Electrical Manufacturers Association; 2001.

24. Watson CC, Casey ME, Eriksson L, Mulnix T, Adams D, Bendriem B. NEMA NU 2 performance tests for scanners with intrinsic radioactivity. J Nucl Med. 2004;45:822-826.

25. Erdi YE, Nehmeh SA, Mulnix T, Humm JL, Watson CC. PET performance measurements for an LSO-based combined PET/CT scanner using the National
Electrical Manufacturers Association NU 2-2001 standard. J Nucl Med. 2004; 45:813-821.

26. Eriksson L, Watson CC, Wienhard K, et al. The ECAT HRRT: an example of NEMA scatter estimation issues for LSO-based PET systems. IEEE Trans Nucl Sci. 2005;52:90-94.

27. Bailey DL, Jones T, Spinks TJ. A method for measuring the absolute sensitivity of positron emission tomographic scanners. Eur J Nucl Med. 1991;18: 374-379.

28. Seidel J, Vaquero JJ, Lee IJ, Green MV. Experimental estimates of the absolute sensitivity of a small animal PET scanner with depth-of-interaction capability. Conference Records IEEE Nuclear Science Symposium and Medical Imaging, Lyon, France, October 15-20, 2000. Piscataway, NJ: IEEE; 2000.

29. Nuyts J, Dupont P, Stroobants S, Maes A, Mortelmans L, Suetens P. Evaluation of maximum-likelihood based attenuation correction in positron emission tomography. IEEE Trans Nucl Sci. 1999;46:1136-1141.

30. Moses WW, Derenzo SE. Empirical observation of resolution degradation on positron emission tomographs utilizing block detectors [abstract]. J Nucl Med. 1993;34(suppl):101P.

31. Tomic N, Thompson CJ, Casey ME. Investigation of the "block effect" on spatial resolution in PET detectors. IEEE Trans Nucl Sci. 2005;52:599-605.

32. International Commission on Radiation Units and Measurements. ICRU Report 37: Stopping Powers for Electrons and Positrons. Bethesda, MD: International Commission on Radiation Units and Measurements; 1984.

33. Wang Y, Seidel J, Tsui BM, Vaquero JJ, Pomper MG. Performance evaluation of the GE Healthcare eXplore VISTA dual-ring small-animal PET scanner. J Nucl Med. 2006;47:1891-1900. 